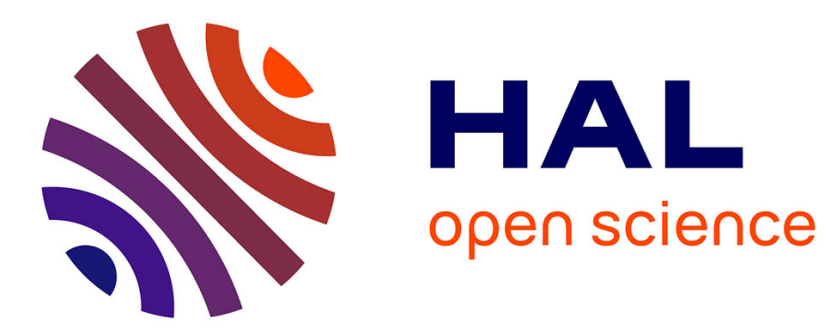

\title{
Capillary-induced phase separation in ultrathin jets of rigid-chain polymer solutions
}

\author{
Andrey V Subbotin, Alexander Semenov
}

\section{To cite this version:}

Andrey V Subbotin, Alexander Semenov. Capillary-induced phase separation in ultrathin jets of rigid-chain polymer solutions. JETP Letters, 2020, 111 (1), pp.55-61. 10.1134/S0021364020010051 . hal-02916110

\section{HAL Id: hal-02916110 \\ https://hal.science/hal-02916110}

Submitted on 17 Aug 2020

HAL is a multi-disciplinary open access archive for the deposit and dissemination of scientific research documents, whether they are published or not. The documents may come from teaching and research institutions in France or abroad, or from public or private research centers.
L'archive ouverte pluridisciplinaire HAL, est destinée au dépôt et à la diffusion de documents scientifiques de niveau recherche, publiés ou non, émanant des établissements d'enseignement et de recherche français ou étrangers, des laboratoires publics ou privés. 


\title{
Capillary-induced phase separation in ultrathin jets of rigid-chain polymer solutions
}

\author{
Andrey V. Subbotin ${ }^{*} \#, \mathbf{1}$ and Alexander N. Semenov ${ }^{* *}$ \\ *Topchiev Institute of Petrochemical Synthesis, Russian Academy of Sciences, Leninskii prosp. \\ 29, Moscow, 119991, Russia \\ ${ }^{\#}$ Frumkin Institute of Physical Chemistry and Electrochemistry, Russian Academy of Sciences, \\ Leninskii prosp. 31, Moscow, 119071, Russia \\ **Institut Charles Sadron, CNRS-UPR 22, Universite de Strasbourg, 23 rue du Loess, BP \\ 84047, 67034 Strasbourg Cedex 2, France
}

Submitted 23 October 2019

In the present paper, we study the terminal stage of the capillary thinning of a polymer solution jet formed with rod-like molecules. On long scales exceeding the rod length a uniform jet gets unstable according to the classical Plateau-Rayleigh pinching mechanism. We show, however, that a qualitatively different faster process, which can prevent the jet from breaking-up, generically comes into play once the jet radius becomes smaller than the rod length. Namely the solvent drains out onto the jet surface forming annular droplets there, while the rods stay trapped inside the jet polymer core. As a result, the jet core becomes more concentrated and can solidify eventually. This process can provide a universal mechanism of the capillary-induced solvent/polymer phase separation leading to fiber formation (fiber spinning) from rod-like polymer solution jets.

\footnotetext{
1 e-mail: subbotin@ips.ac.ru
} 
Capillary thinning and break-up of liquid jets is one of the long-standing important problems attracting wide scientific and industrial interest [1]. The problem is particularly challenging in the case of polymer liquids [2,3]. One relevant application domain of polymer liquids is spinning of fibers which is well known in nature and is widely involved in technological processes like artificial fiber production and electrospinning [4,5]. The physics standing behind the effect is far from being fully uncovered and still presents a formidable issue in spite of a long history of efforts to clarify it.

Last decades the essential progress has been attained in study of Newtonian jets [6,7]. However experiments reveal that Newtonian and polymer liquid threads show qualitatively different types of behavior. It was found that jets formed by solutions of flexible polymers can show formation of periodic beads-on-a-string structures [8,9] and blistering patterns where solvent droplets set on micro- or nano-fibers [10-13]. The beads-on-a-string pattern arises upon capillary pinching and involves spherical beads separated by narrow threads with stretched chains and constant polymer concentration $[13,14]$. The nature of the blistering patterns is less studied. One of the approaches elucidating this phenomenon based on opportunity of polymer molecules migrate in the thinner regions due to the stress-concentration coupling effect $[15,16]$. However, this approach faces difficulties in the analysis of non-linear behavior of polymer jets, as it disregards short-scale modes. Such dynamical modes are captured by the molecular models. One such model has recently been used to study the flow induced phase separation in dilute polymer solutions under extension $[17,18]$. It predicts a polymer/solvent demixing due to a flowinduced orientation of polymer chains acting to reverse their effective interactions from repulsive to attractive. As a result the elongated chains form a network of fibrils which then tends to compress by squeezing out the solvent to the surface. The emerging solvent layer turns then unstable with respect to undulations. This instability can be understood in analogy with that of a liquid annular coating of a wire [19]. In the latter case undulations with the period $\lambda \simeq 2 \pi a \sqrt{2}$ where $a$ is the jet radius have been predicted in good agreement with experiments [10].

Most of the above results cover solutions of flexible chains. Meanwhile solutions of stiff polymers such as polypeptides, DNA, cellulose, aromatic polyamide copolymers etc. are particularly interesting for applications. Such polymer systems show anisotropic phases with high degree of order; they are also capable of forming high performance fibers and play an important role in living systems [20-22]. Our study is focused on capillary thinning of solutions of stiff polymers in the regime of ultrathin jet when its diameter is smaller than the polymer chain length and the chains are highly oriented along the jet axis. Such regime arises at the terminal stage of capillary thinning. Focusing on the jet dynamics for solutions of rod-like 
macromolecules we show that polymer/solvent demixing occurs due to capillary forces, i.e. the capillary forces activate drainage of the solvent to the jet surface and trap rods inside the jet core. It is interesting to note that this mechanism is likely to be qualitatively applicable also for solutions of semiflexible polymers when the chains are highly stretched by the extensional flow.

Let us consider a semidilute solution of rod-like macromolecules of length $L$ and diameter $d, d \ll L$. The average concentration $c$ is such that $c L^{3} \gg 1$ meaning that the rods are crowded while their volume fraction $\phi=\pi L d^{2} c / 4 \ll 1$. The dynamics of an isolated rod in the dilute solution without entanglements is characterized by the rotational diffusion constant $D_{r 0}=6 / \tau_{r}$ where $\tau_{r}$ is the characteristic time necessary for a rod to move on a distance $\sim L$ along its axis, $\tau_{r} \simeq \frac{\zeta_{\|} L^{3}}{k_{B} T}$ [23]. In the last formula $\zeta_{\|}=2 \pi \eta_{s} / k_{H}$ is the parallel component of the friction coefficient per unit rod length, $k_{B}$ is the Boltzmann constant, $T$ is the temperature, $\eta_{s}$ is the viscosity of the solvent and $k_{H}$ is the hydrodynamic factor which for isolated rod equals to $k_{H} \simeq \ln (L / d)$. In the crowded system the dynamics of rods must essentially slow down. According to the Doi and Edwards theory [23] the rotational diffusion of "entangled" rods is characterized by diffusion constant $D_{r} \simeq\left(a_{t} / L\right)^{2} D_{r 0}$, where $a_{t}$ is a characteristic diameter of the tube created by the neighboring rods. For semidilute solutions the hydrodynamic factor reads $k_{H} \simeq \ln \left(\xi_{H} / d\right) \simeq 0.5 \ln (1 / \phi)$ where $\xi_{H} \sim \xi=(c L)^{-1 / 2}$ is the screening length which is of the order of the average distance between the neighboring rods $\xi[18,22]$. This length is roughly the mean distance from an arbitrary point to the nearest rod. The tube diameter for an isotropic solution of rods is estimated as $a_{t} \simeq L \sqrt{\beta} /\left(c L^{3}\right)$ where $\beta \sim 1000$ is a numerical factor [24], therefore the rotation of rods gets hindered if $c L^{3}>\sqrt{\beta}$.

Next we consider formed by the solution uniform cylindrical jet of length $L_{z} \gg L$ and radius $a \ll L_{z}$ in the regime where inertial effects can be neglected. The latter condition is satisfied if $a a^{*} \gg L_{z}^{2}$, where $a^{*}=\eta^{2} / \rho \gamma$ is the characteristic radius separating jets with viscosity dominated and inertia dominated behavior [1]. Here $\rho$ is density of the liquid, $\gamma$ is its surface tension and $\eta$ is its viscosity. Obviously, the jet has to pinch due to capillary forces so that its radius decreases as $a(t) \propto e^{-\dot{\varepsilon} t / 2}$ and the velocity field inside the jet in cylindrical coordinates $(z, r, \varphi)$ is given by $\mathbf{v}_{0}=\dot{\varepsilon} z \mathbf{e}_{z}-\frac{\dot{\varepsilon} r}{2} \mathbf{e}_{r}$ where $\dot{\varepsilon}$ is the elongation rate. Here $\mathbf{e}_{z}$ is the unit vector 
along $z$-axis and $\mathbf{e}_{r}$ is a radial unit vector. Upon stretching the rods get oriented along the z-axis. Their orientational distribution is given by the function $f(\mathbf{n}), \int f(\mathbf{n}) d \Omega_{\mathbf{n}}=1$, where $\mathbf{n}$ is directed along the rod axis and $d \Omega_{\mathbf{n}}$ is an element of the steric angle around the direction $\mathbf{n}$. Rotational diffusion coefficient of the rods in the anisotropic state is given by $\bar{D}_{r} \simeq D_{r} I^{-2}$ with $I=\frac{4}{\pi} \int f\left(\mathbf{n}_{1}\right) f\left(\mathbf{n}_{2}\right)\left|\mathbf{n}_{1} \times \mathbf{n}_{2}\right| d \Omega_{\mathbf{n}_{1}} d \Omega_{\mathbf{n}_{2}}$ [48]. The distribution function $f(\mathbf{n})$ is found from the Smoluchowski equation for rotational motion of the rods and for the elongational flow is given by [23]

$$
f(\mathbf{n})=\text { const } \cdot \exp \left(\frac{3 \dot{\varepsilon}}{4 \bar{D}_{r}} \cos ^{2} \theta\right)=\text { const } \cdot \exp \left(\alpha \cos ^{2} \theta\right)
$$

where $\cos \theta=\mathbf{n e}_{z}$. Parameter $\alpha$ is obtained from Eq. (1) self-consistently. At $\alpha \gg 1$ $(I \sim 1 / \sqrt{\alpha})$ it is written as

$$
\alpha \simeq\left\{\begin{array}{lc}
\left(6 \dot{\varepsilon} \tau_{r} / \pi\right)^{1 / 2}\left(L / a_{t}\right), & \left(a_{t} / L\right)^{2} \ll \dot{\varepsilon} \tau_{r} \ll\left(L / a_{t}\right)^{2} \\
\dot{\varepsilon} \tau_{r} / 8, & \dot{\varepsilon} \tau_{r} \gg\left(L / a_{t}\right)^{2}
\end{array}\right.
$$

Below we consider highly oriented rods when the characteristic value of the angle between the rod axis and $z$-axis is smaller than the magnitude of the angle fluctuations inside the tube,

$$
\langle\theta\rangle \simeq 1 / \sqrt{\alpha}<\left(a_{t} / L\right) \sim \sqrt{\beta} /\left(c L^{3}\right)
$$

It leads to $\alpha>\left(c L^{3}\right)^{2} / \beta$. The condition (3) corresponds to the second line in Eq. (2).

It is well known that the shape of the cylindrical jet is unstable with respect to undulations [1]. We can assess the instability effects rendering the jet non-uniform. The subsequent dynamics of the jet is governed by the capillary forces. It is important however that in ultrathin jet when its radius less than rod length, $a<L$, the dynamics of long-wave fluctuations with the period $\lambda$ exceeding the rod length, $\lambda \gg L$, differs from the dynamics of short-wave fluctuations with $a<\lambda<L$.

In the first regime, $\lambda \gg L>a$, we can employ the conventional approach assuming that the solution of rod-like polymers is approximated by the continuum medium. Concentration of rods in this case is constant. The effective viscosity $\eta$ of the solution can be obtained based on the general equation for the stress tensor $\boldsymbol{\sigma}[23]$ :

$$
\sigma_{i j}=\eta_{s}\left(\kappa_{i j}+\kappa_{j i}\right)-c k_{B} T \delta_{i j}+c k_{B} T\left(3\left\langle n_{i} n_{j}\right\rangle-\delta_{i j}\right)+\frac{c k_{B} T}{2 D_{r 0}}\left\langle n_{i} n_{j} n_{l} n_{m}\right\rangle \kappa_{l m}
$$


Here $\kappa_{i j}=\partial v_{i} / \partial x_{j}$ is the velocity gradient tensor. For elongational flow $\kappa_{z z}=\dot{\varepsilon}$, $\kappa_{x x}=\kappa_{y y}=-\dot{\varepsilon} / 2$. When rods are strongly oriented $\left(n_{z} \approx 1, n_{x} \approx 0, n_{y} \approx 0\right)$ and $\dot{\varepsilon} \gg D_{r 0}$ the normal stress $N=\sigma_{z z}-\sigma_{x x}$ reads

$$
N \simeq 3 \eta_{s} \dot{\varepsilon}+3 c k_{B} T+\frac{c k_{B} T}{2 D_{r 0}} \dot{\varepsilon} \simeq 3 c k_{B} T+3 \eta \dot{\varepsilon}
$$

and the effective viscosity is given by $\eta \simeq \eta_{s}\left(1+\frac{\pi}{18 k_{H}} c L^{3}\right)$. The last formula is similar to that for viscosity of solutions of flexible chains being in the stretched state [12].

The dynamics of the jet in the scope of the slender body approximation $(|\partial a / \partial z| \ll 1)[1]$ is described by the volume conservation equation

$$
\frac{\partial a^{2}}{\partial t}+\frac{\partial}{\partial z}\left(a^{2} v_{z}\right)=0
$$

and by the momentum equation

$$
\frac{\partial}{\partial t}\left(\rho a^{2} v_{z}\right)+\frac{\partial}{\partial z}\left[a^{2}\left(\rho v_{z}^{2}-3 \eta \frac{\partial v_{z}}{\partial z}\right)\right]+\gamma a^{2} \frac{\partial C}{\partial z}=0
$$

Here the radius $a$ and velocity $v_{z}$ depend on the axial coordinate $z$ and time $t . C$ is the total curvature of the jet so that the Laplace pressure is $\gamma C$. Linear stability analysis shows that the fastest growing mode of the jet shape fluctuations is characterized by the wave vector $k=2 \pi / \lambda$ and the growth rate $\Gamma[1]$ :

$$
k=\frac{1}{a_{0}}\left(2+\sqrt{a_{0}^{*} / a_{0}}\right)^{-1 / 2}, \quad \Gamma=\frac{3 \eta}{\rho a_{0}^{2}} \sqrt{a_{0} / a_{0}^{*}}\left(2+\sqrt{a_{0}^{*} / a_{0}}\right)^{-1}
$$

Here $a_{0}^{*}=\frac{18 \eta^{2}}{\rho \gamma}=18 a^{*}$. For example, at $\eta \sim 1 \mathrm{P}, c L^{3} \sim 100, \rho \sim 1 \mathrm{~g} \cdot \mathrm{cm}^{-3}, \gamma \sim 100 \mathrm{erg} \cdot \mathrm{cm}^{-2}$ one gets $a_{0}^{*} \sim 1 \mathrm{~mm}$, i.e. a micron-sized jet falls in the viscosity dominated regime with the characteristic break-up time $\tau^{\#}=\Gamma^{-1}=\frac{6 \eta a_{0}}{\gamma} \sim \frac{\eta_{s} a_{0} L^{2}}{\gamma \xi^{2}} \quad\left(c L^{3} \sim L^{2} / \xi^{2} \gg 1\right)$. The condition $\tau^{\#} D_{r 0} \ll 1$ ensures that the rods are kept highly oriented by the flow during the jet thinning process. In the viscosity dominated regime the condition $L / \lambda \ll 1$ is satisfied if the radius $a_{0} \gg(2 \pi)^{-4 / 3} L\left(L / a_{0}^{*}\right)^{1 / 3}$.

Let us consider the regime of a thin liquid jet of radius smaller than the rod length: $a=a_{0} \ll L$. Below we show that in the short-wave regime, $a_{0}<\lambda<L$, the 
fluctuation growth and consequent variation of the cylindrical shape are driven by a drainage of the solvent to the surface leading to a shrinkage of the system of rods inside the core of radius $a_{-}<a_{0}$ as illustrated in Fig. 1(a,b). As long as rods repel each other, the free energy of the core increases upon its compression thereby hindering the fluctuation growth. On the contrary, the surface energy decreases if the fluctuation width along the $z$-axis exceeds the radius $a_{0}$. Thus, the short-wave pinching dynamics of the jet depends on the balance between the surface and volume forces.

(a)

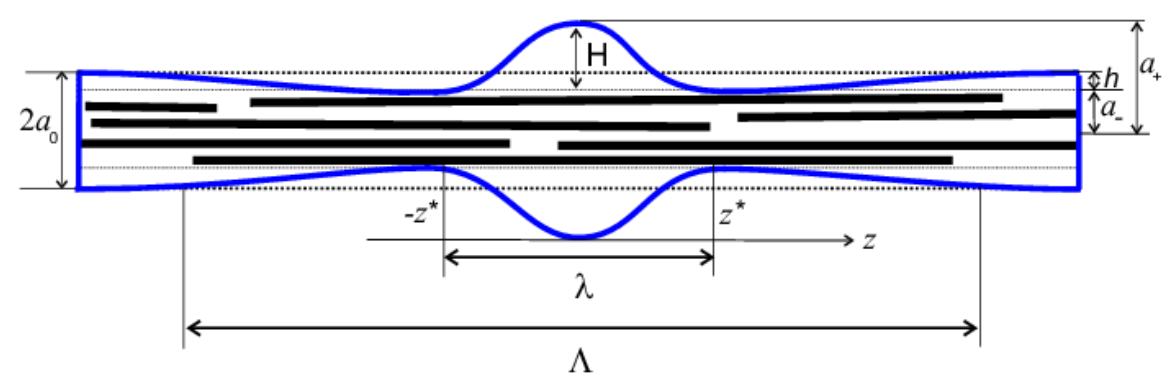

(b)

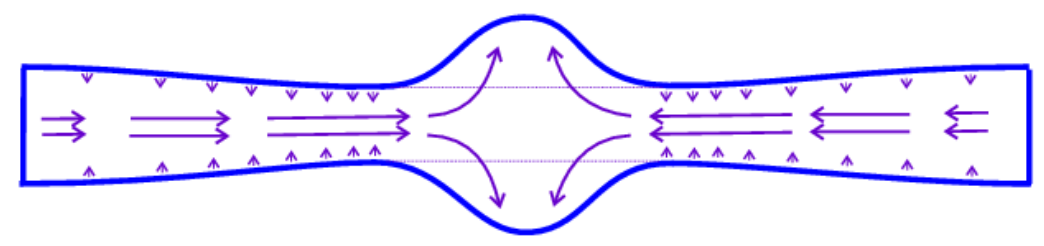

Fig. 1. (a) Illustration of the fluctuation in the short-wave regime. $H=a_{+}-a_{-}$is the annular droplet height, $\lambda$ is its length along the axis, $z^{*}=\lambda / 2, a_{-}$is the radius of the droplet/core interface, $h=a_{0}-a_{-}$is the shrinkage depth, $\Lambda$ is the total length of the fluctuation. (b) Schematic picture of the solvent velocity field upon the droplet formation.

To analyze this process let us consider an axially symmetric fluctuation of the jet defined by the profile $a(z)$, see Fig. 1(a). Of course, the jet radius also depends on time, $a(z, t)$, but $t$ dependence is omitted here and below to simplify the notation. It is assumed (and will be seen later) that the jet radius $a_{0}=a_{0}(t)$ does not essentially change during the fluctuation formation. We assume that the solvent phase in the form of an annular droplet of volume $V_{d}$ is localized in 
the region $-z^{*} \leq z \leq z^{*}$ while the rods are confined inside the core of radius $a_{c}$, so that $a_{c}(z)=a(z)$ outside the region $|z|>z^{*}$ and $a_{c}(z)=a\left(z^{*}\right)=a_{-}$when $|z| \leq z^{*}$. The number of confined rods is $\sim n_{0}=\pi a_{0}^{2} c L \gg 1$ and the total length of the fluctuation is $\Lambda$. We generally expect that $\Lambda \geq L$ since otherwise the core volume inaccessible for the centers of aligned rods becomes too large leading to a considerable increase of the osmotic free energy (due to a decrease of the ideal-gas entropy of rods). Therefore the length $\Lambda$ must be much longer than the jet thickness, $\Lambda \gg a_{0}$. At large distances, $|z|>\Lambda / 2$, the core is transformed in the thread of radius $a_{0}$. The droplet volume $V_{d}$ is defined by the volume conservation equation $V_{d}=V_{c} \sim \pi\left(a_{0}^{2}-a_{-}^{2}\right) \Lambda$, where $V_{c}$ is the core volume decrement. The variation of the liquid surface energy is approximately given by

$$
F_{S}=\gamma \Delta A, \quad \Delta A \simeq A_{d}+\int_{z^{*}<|z|<\Lambda / 2} 2 \pi a_{c}(z) d z-2 \pi a_{0} \Lambda
$$

Here $A_{d}=2 \pi \int_{-z^{*}}^{z^{*}} a(z) \sqrt{1+a_{z}^{\prime 2}} d z$ is the free surface area of the droplet and $a_{z}^{\prime}=d a / d z$. Below we assume a weak fluctuation $\left|a(z)-a_{0}\right| \ll a_{0}$, so that the osmotic pressure, $\Pi$, inside the core just slightly increases upon its compression, and the osmotic free energy can be approximated as

$$
F_{V}=V_{d} \Pi
$$

where the osmotic pressure inside the jet is [21],

$$
\Pi \simeq c k_{B} T\left(1+\frac{L \phi}{d} \frac{I(\alpha)}{1-\phi}-\frac{L \phi}{d} \frac{T_{\Theta}}{T}\right)
$$

Here the second term in brackets is due to steric repulsion of rods and $T_{\Theta}$ is the $\Theta$-temperature of the isotropic solution. Note that $c=1 /\left(L \xi^{2}\right)$. For highly oriented rods $I(\alpha) \sim 1 / \sqrt{\alpha} \sim \theta^{*}$ where $\theta^{*}$ is a small typical angle between a rod and $z$-axis, $\theta^{*} \ll 1$. For $\theta^{*} \leq d / L$ the rods become nearly parallel, and the steric effect decouples from the rod orientations. In this regime the osmotic pressure is still defined in eq. (10) provided that $I(\alpha)$ is formally set to $4 d / L$.

The optimum shape of the droplet is found from minimization of the surface area $A_{d}$ at fixed radius of the core and volume of the droplet. It corresponds to the surface of constant curvature $C[24]$, defined by the differential equation

$$
a_{+} a_{-}+a^{2}=\frac{2 a}{C \sqrt{1+a_{z}^{\prime 2}}}, \quad a_{+}+a_{-}=2 / C
$$


where $a=a(z)$ and $a_{+}$defines the height (thickness) $H$ of the solvent droplet: $H=a_{+}-a_{-}$(cf. Fig. 1(a)). Below we assume for simplicity that the radius $a_{-}$of the core/droplet interface is constant; this assumption is justified by the condition $\lambda \ll L$. The solution of Eq. (11a) is presented in the implicit form $\left(a_{-} \leq a \leq a_{+}\right)$

$$
z(a)=\left[\begin{array}{cc}
-z^{*}+\int_{a_{-}}^{a} d a \frac{a_{+} a_{-}+a^{2}}{\sqrt{\left(a_{+}^{2}-a^{2}\right)\left(a^{2}-a_{-}^{2}\right)}}, & -z^{*} \leq z \leq 0 \\
\int_{a}^{a_{+}} d a \frac{a_{+} a_{-}+a^{2}}{\sqrt{\left(a_{+}^{2}-a^{2}\right)\left(a^{2}-a_{-}^{2}\right)}}, & 0 \leq z \leq z^{*}
\end{array}\right.
$$

The droplet profiles for different values of $H$ are shown in Fig. 2.

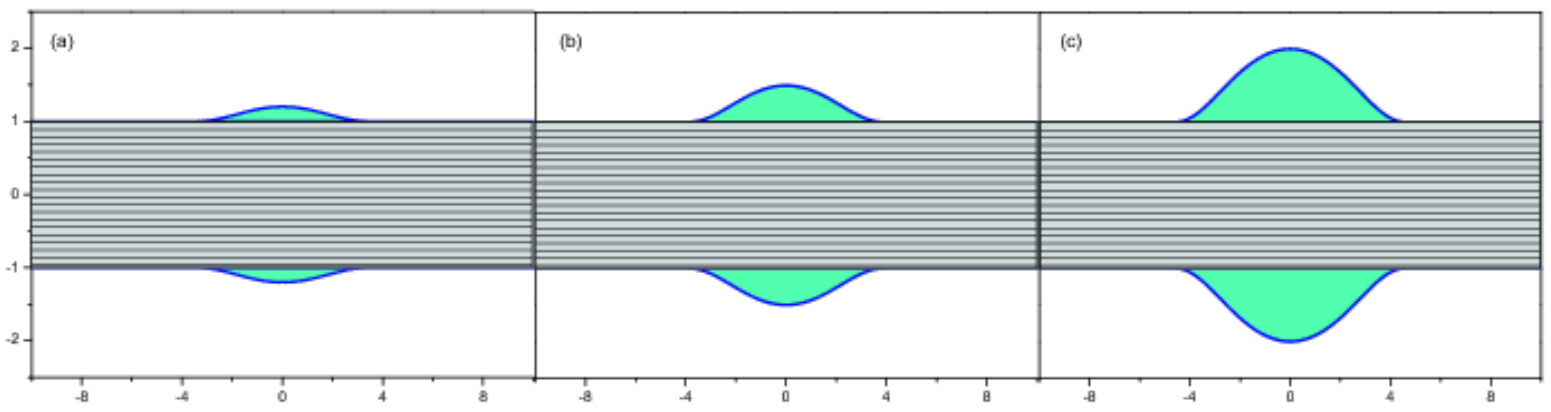

Fig. 2. Annular droplet profiles on the jet core of radius $a_{-}=1$ and (a) $H=0.2$, (b) $H=0.5$ and (c) $H=1$.

The length of the annular droplet is found from eq. (11b) using a new variable $\varphi$ which is defined through the formula $a^{2}=\left[\left(a_{+}^{2}+a_{-}^{2}\right)+\left(a_{+}^{2}-a_{-}^{2}\right) \sin \varphi\right] / 2$ :

$$
\lambda=2 z^{*}=\frac{2}{C} \int_{-\pi / 2}^{\pi / 2} d \varphi \frac{1+\left(1-a_{-} C\right) \sin \varphi}{\sqrt{a_{-}^{2} C^{2}+2\left(1-a_{-} C\right)(1+\sin \varphi)}}
$$

and the surface area and the volume of the annular droplet are written as

$$
\begin{gathered}
A_{d}=\frac{4 \pi}{C^{2}} \int_{-\pi / 2}^{\pi 2} d \varphi \sqrt{a_{-}^{2} C^{2}+2\left(1-a_{-} C\right)(1+\sin \varphi)} \\
V_{d}=\pi \int_{-z^{*}}^{z^{*}}\left(a(z)^{2}-a_{-}^{2}\right) d z=\frac{2 \pi}{C^{3}} \int_{-\pi / 2}^{\pi 2} d \varphi\left(1+\left(1-a_{-} C\right) \sin \varphi\right) \sqrt{a_{-}^{2} C^{2}+2\left(1-a_{-} C\right)(1+\sin \varphi)}-\pi a_{-}^{2} \lambda
\end{gathered}
$$

The droplet surface curvature is represented as $C=\frac{1}{a_{-}}+\Delta C$, where the increment $\Delta C \simeq-\frac{H}{2 a_{0}^{2}}$ for $H \ll a_{0}$ (hence $h \ll a_{0}$ since $h \ll H:$ the relationship between $H$ and $h$ is found from the 
volume conservation equation $\left.V_{d} \simeq 2 \pi^{2} a_{0}^{2} H \sim 2 \pi h a_{0} \Lambda, H \sim \frac{\Lambda h}{\pi a_{0}} \gg h\right)$. Taking into account that $\Delta A \simeq-\frac{\pi^{2} H^{2}}{2}$ (note, $d(\Delta A)=C d V_{d}-C_{c} d V_{c}=\left(C-C_{c}\right) d V_{d} \simeq-\pi^{2} d\left(H^{2} / 2\right)$, where $C_{c} \simeq 1 / a_{-}$is the total curvature of the core surface) the total free energy of the annular droplet reads

$$
F_{S}+F_{V} \simeq-\frac{\pi^{2} \gamma}{2} H^{2}+2 \pi^{2} a_{0}^{2} H \Pi
$$

Maximization of the droplet free energy with respect to $H$ gives the activation state with $H=H^{*} \simeq 2 \Pi a_{0}^{2} / \gamma$. Note that $H=H^{*}$ corresponds to the saddle of the free energy with minimal surface energy for the given droplet volume $V_{d}=V_{d}\left(H^{*}\right)$.

In what follows we neglect the interaction part of the osmotic pressure given by the 2nd and the 3rd terms in Eq. (10). As for the last term, we disregard attraction of rods assuming that it is dominated by their repulsion. Further, the repulsive contribution is dominated by the idealgas contribution, $\Pi \simeq \Pi_{i d} \simeq k_{B} T /\left(L \xi^{2}\right)$ in the regime defined by the condition (15b) below. Thus, using the following estimates: $T \approx 300 \mathrm{~K}, \quad \xi \sim 10 \mathrm{~nm}, L \sim 1 \mu \mathrm{m}, \quad a_{0} \sim 100 \mathrm{~nm}$, $\gamma \sim 100 \mathrm{erg} \cdot \mathrm{cm}^{-2}$ one finds $H^{*} / a_{0} \simeq 2 \Pi a_{0} / \gamma \sim 10^{-4}$, hence $H^{*} \ll a_{0}$. The droplet activation energy (the energy barrier separating the regimes of stable and unstable droplet) is

$$
F_{b a r r} \simeq \frac{\pi^{2}}{2} \gamma H^{* 2} \simeq 2 \pi^{2} \Pi^{2} a_{0}^{4} / \gamma
$$

Using $\Pi \sim \Pi_{i d}$ we get (also using the above estimates of $L$ and $\gamma$ ):

$$
\frac{F_{\text {barr }}}{k_{B} T} \simeq 2 \pi^{2} \frac{k_{B} T}{\gamma L^{2}} \frac{a_{0}^{4}}{\xi^{4}} \sim 10^{-6}\left(\frac{a_{0}}{\xi}\right)^{4}
$$

The reduced activation energy thus depends strongly on the ratio $a_{0} / \xi$. It becomes significant $\left(F_{\text {barr }} / k_{B} T \geq 1\right)$ for $a_{0} / \xi \geq 30$.

Below we will analyze the case $F_{b a r r} \gg k_{B} T$ when only a single droplet is formed. We also assume that the fluctuation of length $\Lambda \sim L$. This choice can be justified for the critical nucleation stage of droplet formation, $H \sim H^{*}$ (see Fig. 1(a)): fluctuations with $\Lambda \gg L$ are too slow because the relevant solvent/rod friction force is proportional to $\Lambda$, while the osmotic energy penalty for droplet nucleation strongly increases in the regime $\Lambda \ll L$. The characteristic time of the optimum fluctuation formation is estimated as $\tau^{*} e^{\frac{F_{\text {barr }}}{k_{B} T}}$ where $\tau^{*}$ is the time which is 
required to create a critical droplet of volume $V_{d} \sim a_{0}^{2} H^{*}$, i.e. $\tau^{*} \sim \frac{V_{d}}{w_{s z} a_{0}^{2}} \sim \frac{H^{*}}{w_{s z}}$. Here $w_{s z}$ is $z-$ component of the solvent velocity inside the core (solvent moves in the longitudinal direction from periphery into the forming droplet, Fig. 1(b)). This velocity can be found by balancing the activation energy and the total energy dissipated due to solvent/rod friction force while the system descends from the barrier. The dissipation rate inside the volume $\sim \pi a_{0}^{2} L$ of the core where the flow is mainly localized is estimated as $\dot{\mathcal{D}} \sim c L \zeta_{\|} w_{s z}^{2} a_{0}^{2} L$, therefore the total amount of dissipated energy during the time $\tau^{*}$ is $\mathcal{D} \sim \dot{\mathcal{D}} \tau^{*} \sim \eta_{s} w_{s z} a_{0}^{2} L H^{*} / \xi^{2}$. Using the relationship $F_{\text {barr }} \sim \mathcal{D}$ one finds $w_{s z} \sim \frac{\gamma^{\xi^{2}} H^{*}}{\eta_{s} a_{0}^{2} L}$ and $\tau^{*} \sim \frac{\eta_{s} a_{0}^{2} L}{\gamma^{2}}$. Obviously, the time $\tau^{*}$ is much shorter than the Plateau-Rayleigh time $\tau^{\#}: \tau^{*} \sim \frac{\eta_{s} a_{0}^{2} L}{\gamma \xi^{2}} \ll \tau^{\#} \sim \frac{\eta_{s} a_{0} L^{2}}{\gamma \xi^{2}}$. Therefore, the droplet formation is faster than $\tau^{\#}$ if $F_{b a r r}<k_{B} T \ln \left(L / a_{0}\right)$.

We can now specify the regime where virial interactions of rods do not alter much the osmotic pressure $\Pi$. For $\phi \ll 1$ (low volume fraction of rods, $\phi \sim c L d^{2}$ ) and $\theta \ll 1$ (highly oriented rods) the relevant condition is (cf. Eq. (10))

$$
\frac{L}{d} \theta \phi \sim L^{2} d c \theta \sim \theta L d / \xi^{2} \ll 1
$$

The angle $\theta$ can be estimated as the typical angle of a rod rotation during the characteristic time $\tau^{*}$. To get an upper bound estimate we assume a free rotation, so $\theta^{2} \sim D_{r 0} \tau^{*}$ leading (with $\left.D_{r 0} \sim k_{B} T /\left(\eta L^{3}\right)\right)$ to $\theta \sim \sqrt{\frac{k_{B} T}{\gamma}} \frac{a_{0}}{L \xi}$. Thus, the condition (15a) is certainly satisfied if

$$
d \ll \frac{\xi^{3}}{a_{0}} \sqrt{\frac{\gamma}{k_{B} T}}
$$

With $\xi \sim 10 \mathrm{~nm}, a_{0} \sim 100 \mathrm{~nm}, \gamma \sim 100 \mathrm{erg} \cdot \mathrm{cm}^{-2}$ we get $d \ll 50 \mathrm{~nm}$, which is a reasonable condition as we expect the rod diameter $d<5 \mathrm{~nm}$.

In the above analysis we assumed that the droplet has an equilibrium shape. It means that the droplet relaxation time $\tau_{d}$ is smaller than $\tau^{*}$. To estimate $\tau_{d}$ let us consider slightly nonequilibrium droplet of curvature $C=\frac{1}{a_{-}}-\frac{H}{2 a_{0}}+\Delta C(z)$ where $\Delta C \sim \frac{H_{1}}{a_{0}^{2}}$. Here $H_{1}$ is the deviation of the droplet height from the equilibrium value: for example the droplet height may be 
of the order of $H^{*}+H_{1}$ in $-z^{*}<z<0$ and $H^{*}-H_{1}$ in $0<z<z^{*}$. The surface energy excess in this case is $\Delta F \sim \gamma H_{1}^{2}$ and the pressure gradient along the z-axis is $P^{\prime} \sim \gamma \frac{\Delta C}{\lambda} \sim \frac{\gamma H_{1}}{a_{0}^{3}}$. The pressure gradient generates a flow both inside the droplet and inside the core. The characteristic longitudinal velocity inside the droplet and the core is denoted as $v_{d z}$ and $v_{c z}$ correspondingly. The relaxation time is the ratio of the half droplet volume deviation $\sim a_{0}^{2} H_{1}$ from the equilibrium value to the total flux $J$ which is a sum of the flux inside the droplet, $\sim a_{0} H v_{d z}$, and the flux inside the core $\sim a_{0} \ell v_{c z}: \tau_{d} \sim a_{0}^{2} H_{1} / J, J \sim a_{0} H v_{d z}+a_{0} \ell v_{c z}$, where $\ell$ is the flow penetration depth in the core. The velocity $v_{d z}$ of the flow inside the droplet is found from hydrodynamic equation $\eta_{s} \frac{d^{2} v_{d z}}{d r^{2}} \sim P^{\prime}$, hence $\eta_{s} \frac{v_{d z}}{H^{2}} \sim \frac{\gamma H_{1}}{a_{0}^{3}}$ and $v_{d z} \sim \frac{\gamma H^{2} H_{1}}{\eta_{s} a_{0}^{3}}$. The balance between the pressure gradient and the friction force inside the core reads $c L \zeta_{\|} v_{c z} \sim \frac{\gamma H_{1}}{a_{0}^{3}}$, i.e. $v_{c z} \sim \frac{\gamma \xi^{2} H_{1}}{\eta_{s} a_{0}^{3}}$. The depth $\ell$ can be obtained based on the principle of maximum dissipation rate [25]. The energy dissipated during the time $\tau_{d}$ is $\Delta F$, hence the dissipation rate is of the order of $\Delta F / \tau_{d}$. Its maximum is attained when $\ell$ is maximized: $\ell \sim a_{0}$. Hence

$$
\tau_{d} \sim\left[\begin{array}{ll}
\frac{\eta_{s} a_{0}^{3}}{\gamma \xi^{2}}, & H^{3}<a_{0} \xi^{2} \\
\frac{\eta_{s} a_{0}^{4}}{\gamma H^{3}}, & H^{3}>a_{0} \xi^{2}
\end{array}\right.
$$

Obviously, $\tau_{d} \ll \tau^{*}$ as $a_{0} \ll L$.

Once the potential barrier is overcome the droplets start to grow while the core shrinks due to capillary forces. The dynamics of the droplet growth can be found based on the energydissipation balance equation

$$
d F_{S} / d t=-\dot{\mathcal{D}}
$$

Here $F_{S} \sim-\gamma H^{2}$ is the surface energy increment and $\dot{\mathcal{D}} \sim c L \zeta_{\|} w_{s z}^{2} a_{0}^{2} L \sim w_{s z}^{2} \tau^{*}$ is the dissipation rate, where $w_{s z}$ is the characteristic solvent velocity inside the core. Hence

$$
d H / d t \sim w_{s z}^{2} \tau^{*} / H
$$

The flow velocity $w_{s z}$ can be found through the balance between the pressure gradient inside the core $\sim \frac{\gamma H}{a_{0}^{2} L}$ and the friction force per unit volume $c L \zeta_{\|} w_{s z}:$ the relationship $\frac{\zeta_{\|} w_{s z}}{\xi^{2}} \sim \frac{\gamma H}{a_{0}^{2} L}$ yields 
$w_{s z} \sim H / \tau^{*}$. After substitution of the last formula in (16b) we arrive at the exponential growth law for the droplet height

$$
H(t) \simeq H^{*} e^{t / \tau^{*}}
$$

Therefore, the droplet thickness becomes of the order of the jet radius, $H \simeq a_{0}$, in a short growth time $t_{1}=\tau \ln \left(a_{0} / H^{*}\right) \sim \tau^{*} \ll \tau^{\#}$. The subsequent dynamics of the solvent/polymer phase demixing involves formation of multiple droplets. This regime will be considered separately.

In summary, we examined two qualitatively different mechanisms of polymer liquid jet instability arisen on long and short length-scales. We focused on the most interesting regime when the jet thickness is smaller than the rod length and the rods are highly oriented. For lengthscales exceeding the rod length the surface tension-driven thinning develops in a conventional way (Plateau-Rayleigh mechanism [1]) which ultimately should result in breaking up of the jet. By contrast, we show that at shorter length-scales the rods get effectively trapped inside the jet core whereas the solvent drains to the surface and forms droplets there. The latter process of capillary phase separation occurs much faster and can prevent the jet from breaking up. This mechanism works both with non-volatile solvents and with no specific attraction between oriented polymer chains and differs from the mechanism of phase separation which is connected with a reduction of the steric repulsion of the stretched chains [17,18]. Moreover, the described mechanism may be also at work in solutions of semiflexible polymers if the chains are highly stretched due to extension. Thereby the discovered capillary-driven phase separation effect can provide a universal mechanism of fiber formation in solutions of stretched polymers.

Experimental observation and identification of the predicted solvent/polymer demixing mechanism is a challenging problem. A thin quasi-uniform jet can be easily produced, for example, by stretching of a liquid droplet. This process gives rise to formation of a thin neck whose length $L_{z}=L_{z}(t)$ and radius $a=a(t)$ decrease in time. The theory developed above is applicable to such pinching bridge with $a \ll L_{z}$ which can be locally considered as a uniform cylinder. The jet radius $a$ must be small enough, $a \ll a^{*}$, where $a^{*}=\eta^{2} / \rho \gamma$, and $L_{z} \ll \sqrt{a a^{*}}$. Quite obviously, the polymer solutions should contain very long macromolecules which are aligned by the flow. One option is to use solutions of DNA. These chains can be very long and form nano-thick fibers in solutions [26]. The most appropriate system to verify our results must involve very long rods. Such macromolecular rods (with length $\geq 1-10$ microns ) are known, examples are given by protein polymers (F-actin, microtubules) and other self-assembling supramolecular structures (like tri-arylamine fibers) [27-31]. 
A.V.S. acknowledges financial support from the Russian Science Foundation (Grant \# 1779-30108). A.N.S. acknowledges a partial support from the International Research Training Group (IRTG) "Soft Matter Science: Concepts for the Design of Functional Materials".

1. J. Eggers and E. Villermaux, Rep. Prog. Phys. 71, 036601 (2008).

2. G. H. McKinley, Rheol. Rev. 1 (2005).

3. A.Ya. Malkin, A. Arinstein, and V.G. Kulichikhin, Progr. Polym. Si. 39, 959 (2014).

4. J. Xue, T. Wu, Y. Dai, and Y. Xia, Chem. Rev. 119, 5298 (2019).

5. V. G. Kulichikhin,, I. Yu. Skvortsov, A. V. Subbotin, S. V. Kotomin, and A. Ya. Malkin, Polymers 10, 856 (2018).

6. J. R. Castrejón-Pita, A. A. Castrejón-Pita, S. S. Thete, K. Sambath, I. M. Hutchings, J. Hinch, J. R. Lister, and O. A. Basaran, PNAS, 112, 4582 (2015).

7. Y. Li and J.E.Sprittles, J. Fluid Mech. 797, 29 (2016).

8. A. V. Bazilevskii, S.I. Voronkov, V.M.Entov, and A.N. Rozhkov, Sov. Phys. Dokl., 26, 333 (1981).

9. C. Clasen, J. Eggers, M. A. Fontelos, J. Li, and G. H. McKinley, J. Fluid Mech. 556, 283 (2006).

10. R. Sattler, S. Gier, J. Eggers, and C. Wagner, Phys. Fluids 24, 023101 (2012).

11. A. V. Semakov, I. Yu. Skvortsov, V. G. Kulichikhin, and A. Ya. Malkin, JETP Lett, 101, 690 (2015).

12. A. Ya Malkin, A. V. Semakov, I. Yu. Skvortsov, P. Zatonskikh, V. G. Kulichikhin, A. V. Subbotin, and A. N. Semenov, Macromolecules 50, 8231 (2017).

13. H.-C. Chang, E. A. Demekhin, and E. Kalaidin, Phys. Fluids 11, 1717-1737 (1999).

14. P. P. Bhat, S. Appathurai, M. T. Harris, M. Pasquali, G. H. McKinley, and O. A. Basaran, Nat. Phys. 6, 625 (2010).

15. J. Eggers, Phys. Fluids 26, 033106 (2014).

16. M. Doi and A. Onuki, J. Phys. II France 2, 1631 (1992).

17. A.V. Subbotin and A.N. Semenov, J. Polym. Sci.: Part B: Phys. Ed. 54, 1066 (2016).

18. A.N. Semenov and A.V. Subbotin, J. Polym. Sci.: Part B: Phys. Ed. 55, 623 (2017).

19. S. P. Papkov and V. G. Kulichikhin, The Liquid-Crystalline State of Polymers. (Khimiya, Moscow, 1977).

20. A.N. Semenov and A.R. Khokhlov, Phys. Usp. 156, 988 (1988).

21. Afshari M., Sikkema D. J., Lee K., and M. Bogle, Polym. Rev., 48, 230 (2008). 
22. M. Doi and S. F. Edwards, The Theory of Polymer Dynamics. (Oxford Univ. Press, New York, 1986).

23. I. Teraoka, N. Ookubo, and R. Hayakawa, Phys. Rev. Lett. 55, 2712 (1985).

24. E. A. Maresov and A. N. Semenov, Macromolecules 41, 9439 (2008).

25. L.M. Martyushev and V.D. Seleznev, Physics Reports 426, 1 (2006).

26. X. Fang and D. H. Reneker, J. Macromol. Sci.: Part B: Phys., 36, 169 (1997).

27. F. Gittes, B. Mickey, J. Nettleton, and J. Howard, J. Cell Biology 120, 923 (1993).

28. F. Oosawa and S. Asakura, Thermodynamics of the Polymerization of Protein. (Acad. Press, San Diego, 1975).

29. J. van Mameren, K. C. Vermeulen, F. Gittes, and C. F. Schmidt, J. Phys Chem B, 113, 3837 (2009).

30. E. Moulin, F. Niess, M. Maaloum, E. Buhler, I. Nyrkova, and N. Giuseppone, Angew. Chem. Int. Ed., 49, 6974 (2010).

31. I. Nyrkova, E. Moulin, J. J. Armao IV, M. Maaloum, B. Heinrich, M. Rawiso, F. Niess, J.J Cid, N. Jouault, E. Buhler, A. N. Semenov, and N. Giuseppone, ACS Nano, 8, 10111 (2014). 